\title{
Ground glass opacity featured lung adenocarcinoma in teenagers
}

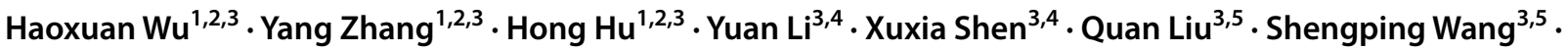 \\ Haiquan Chen ${ }^{1,2,3}$ (1)
}

Received: 1 January 2021 / Accepted: 20 March 2021 / Published online: 7 April 2021

(C) The Author(s), under exclusive licence to Springer-Verlag GmbH Germany, part of Springer Nature 2021

\begin{abstract}
Introduction Ground glass opacity (GGO) nodules were found incidentally by computed tomography (CT) scan in some teenagers, which turned out to be lung cancer. The purpose of this study is to summarize the characteristics of teenage patients with GGO featured lung adenocarcinoma.

Methods Patients aging from 13 to 20 who were incidentally diagnosed with lung cancer were reviewed between February 2015 to December 2020. The clinical, radiological and pathological characteristics were analyzed.

Results Totally 12 patients were included. All of them were diagnosed as GGO featured lung cancer through CT scan, with no presenting symptom. The median surveillance before surgery was 5.5 months, and none of these GGO lesions enlarged or altered in the property during the surveillance. The mean tumor diameter was $0.93 \pm 0.25 \mathrm{~cm}$. Ten patients underwent wedge resection by video-assisted thoracoscopic surgery (VATS), 9 of which were minimally invasive adenocarcinoma (MIA) and 1 of which were invasive adenocarcinoma (IAC) in the pathological analysis. One patient underwent VATS leftupper sublobectomy, pathologically diagnosed as MIA and 1 patient underwent VATS left-upper lobectomy with systematic mediastinal lymphadenectomy, pathologically diagnosed as IAC. The median postoperative hospital stay was 3 days. All patients survived without recurrence during a median follow-up of 12.5 months.

Conclusions GGO nodules could be a sign of early-stage teenage lung adenocarcinoma. We proposed a screening strategy with long intervals based on a baseline CT scan for the teenage population, and a treatment strategy for diagnosed teenage patients.
\end{abstract}

Keywords Ground glass opacity nodules $\cdot$ Teenager $\cdot$ Adenocarcinoma $\cdot$ Screening $\cdot$ Treatment

\section{Abbreviations}

GGO Ground-glass opacity

CT Computed tomography

VATS Video-assisted thoracoscopic surgery

Haoxuan $\mathrm{Wu}$ and Yang Zhang contributed equally to this work

Haiquan Chen

hqchen1@yahoo.com

1 Department of Thoracic Surgery and State Key Laboratory of Genetic Engineering, Fudan University Shanghai Cancer Center, 270 Dong-An Road, Shanghai 200032, China

2 Institute of Thoracic Oncology, Fudan University, Shanghai 200032, China

3 Department of Oncology, Shanghai Medical College, Fudan University, Shanghai 200032, China

4 Department of Pathology, Fudan University Shanghai Cancer Center, Shanghai 200032, China

5 Department of Radiology, Fudan University Shanghai Cancer Center, Shanghai 200032, China
LDCT Low-dose computed tomography

BMI Body mass index

MIA Minimally invasive adenocarcinoma

IAC Invasive adenocarcinoma

\section{Introduction}

With the COVID-19 pandemic, chest computed tomography (CT) scans are adopted as a procedure to help diagnose COVID-19 infection. Teenagers suspected of COVID-19 infection have undergone low-dose computed tomography (LDCT). Some ground-glass opacity (GGO) nodules were incidentally detected on CT scans. Surgical resections were performed on those that were highly suspected of malignance, and pathological analysis confirmed that they were malignant tumors. Because lung cancer is considered to be a disease that occurs mainly in the elderly and teenagers are non-high-risk population for 
lung cancer, there is no plan of examination on teenagers during non-pandemic periods. Lack of attention has been paid to teenage lung cancer patients. Thus, we summarized the characteristics of patients aging from 13 to 20 who were incidentally diagnosed as lung cancer, and these lung cancers all presented as GGO nodules in radiology.

\section{Methods}

The study was carried out by searching the Fudan University Shanghai Cancer Center database for patients whose age ranged from 13 to 20 and incidentally diagnosed lung cancer. Eligible patients underwent curative surgery at the Department of Thoracic Surgery, Fudan University Shanghai Cancer Center, between February 2015 to December 2020. The following variables were collected: sex, age, smoking history, cancer family history, body mass index (BMI), surveillance time frame, surgery type, pathology, tumor diameter, pathological stage and postoperative hospital stay. The evaluation of radiology and pathology can refer to the method part of our previous study (Fu et al. 2019). Informed consent of the included patients was waived because the study was retrospective.

\section{Results}

Totally 12 lung cancer patients who were incidentally diagnosed with lung cancer were identified. Figure 1 shows the time distribution of the number of patients enrolled in the study and the proportion of patients enrolled in the study as a percentage of the total number of patients with GGO featured lung cancer undergoing surgery in our center [2 $(0.21 \%), 2(0.15 \%), 3(0.23 \%)$ and $5(0.35 \%)$ patients in the year of 2015, 2018, 2019 and 2020, respectively]. The characteristics of each patient enrolled were shown on Table 1 . There were 5 males and 7 females. The average age was $17.33 \pm 2.19$ years, and average body mass index (BMI) was $21.93 \pm 3.34$. Only one of them had the habit of smoking, half a pack a day for half a year. Three of 12 patients had cancer family history, but none of their family members suffered from lung cancer. None of them had presenting symptoms when diagnosed. The median surveillance time frame before surgery was 5.5 months (ranging from 1 to 12 months). All cases were featured as GGO nodules on CT scan (Fig. 2), and none of them enlarged or altered in the property during the surveillance period.

Ten patients underwent wedge resection by video-assisted thoracoscopic surgery (VATS), 9 of which were pathologically diagnosed as stage Ia1 minimally invasive adenocarcinoma (MIA) and 1 of which were pathologically diagnosed

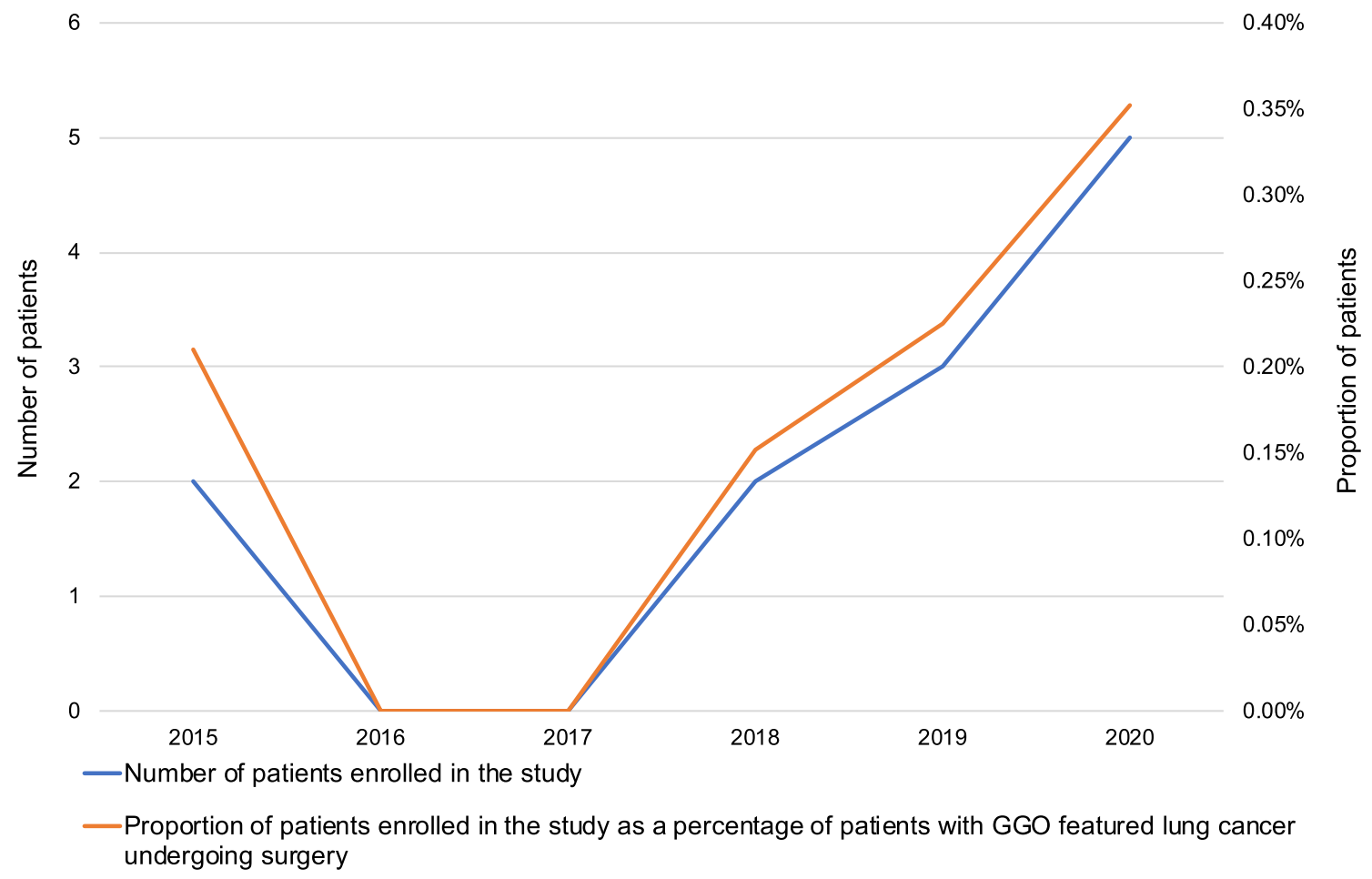

Fig. 1 The time distribution of the number of patients enrolled in the study and the proportion of patients enrolled in the study as a percentage of patients with GGO featured lung cancer undergoing surgery 


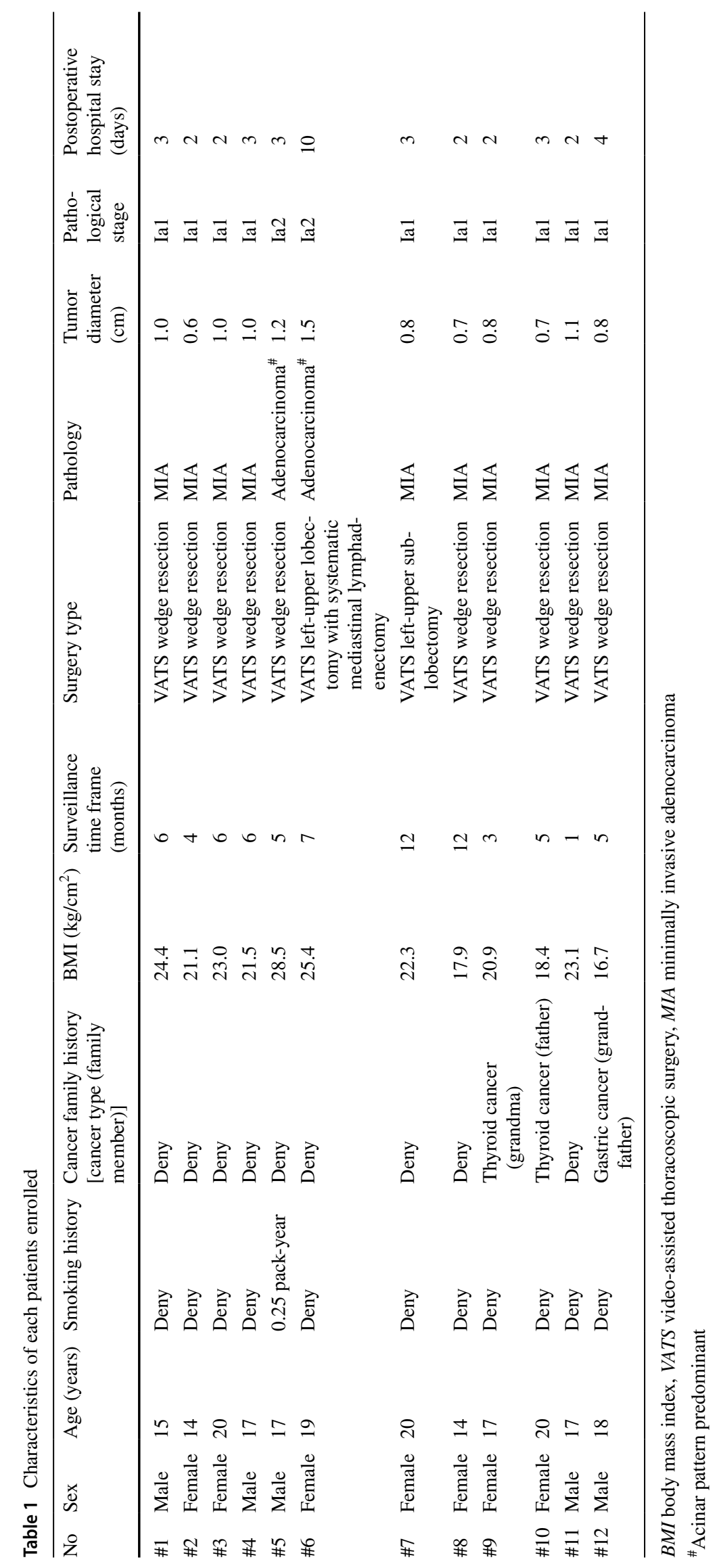




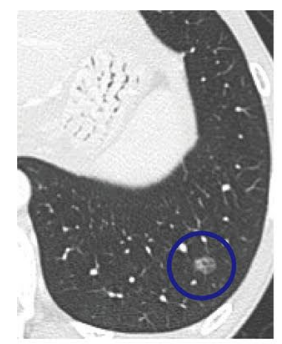

\#1

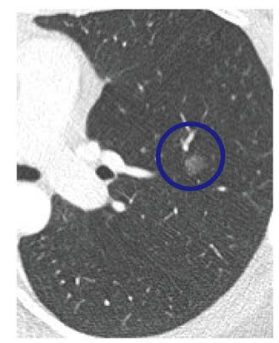

\#7

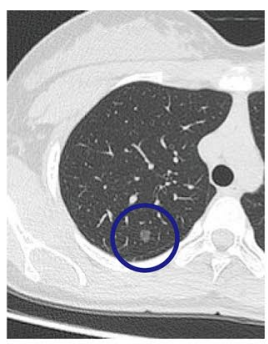

\#2

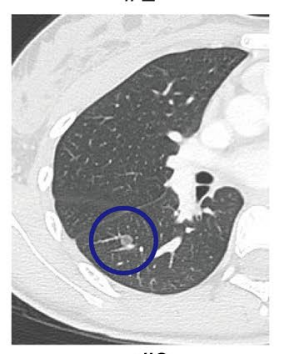

\#8

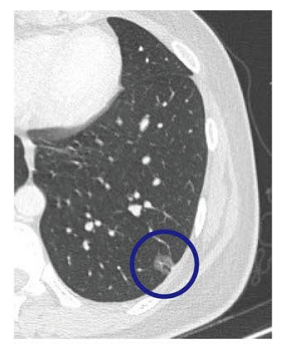

\#3

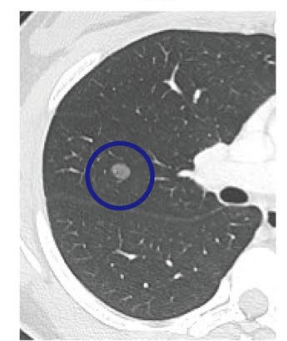

\#9

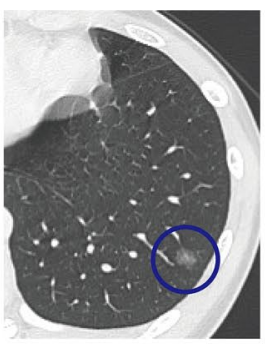

\#4

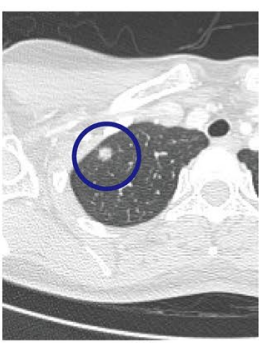

\#10

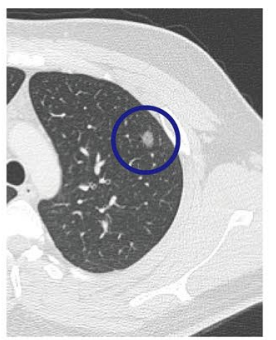

\#5

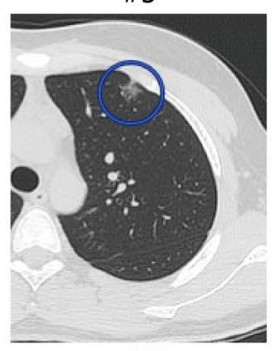

\#11

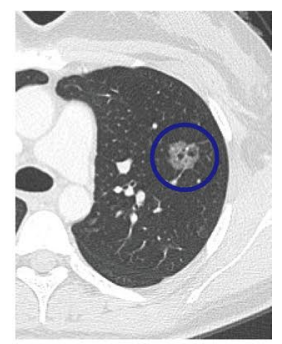

\#6

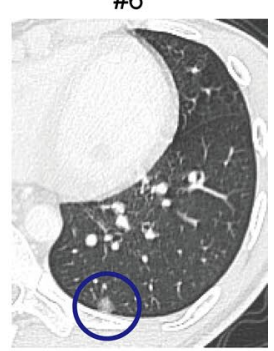

\#12

Fig. 2 The CT images of 12 patients enrolled in the study, listed from \#1 to \#12

as stage Ia2 invasive adenocarcinoma (IAC, acinar pattern predominant). One patient underwent VATS left-upper sublobectomy, pathologically diagnosed as stage Ia1 MIA. One patient underwent VATS left-upper lobectomy with systematic mediastinal lymphadenectomy, pathologically diagnosed as stage Ia2 IAC (acinar pattern predominant). The average pathological tumor diameter was $0.93 \pm 0.25 \mathrm{~cm}$. The median postoperative hospital stay was 3 days (ranging from 2 to 10 days). All patients survived without recurrence, with a median follow-up time of 12.5 months.

\section{Discussion}

In recent years, it has been found that young women and non-smokers had an increased risk for lung cancer (Luo et al. 2017; Jemal et al. 2018). According to a study based on data from regular health examination of Chinese hospital employees, the LDCT detection rate of lung cancer was $1.0 \%$ among people under 40 years old (Zhang et al. 2020). The risk of lung cancer in teenage patients has been rarely discussed. There were a few case reports and case series reviewing teenage lung cancer patients presented as dyspnea or cough, with a large solid mass appeared in CT images (Polo et al. 2015; Emori et al. 1999; Tajiri et al. 1999; Balzer et al. 2018). Majority of the adenocarcinoma patients had metastatic disease at diagnosis and more than half of the patients had died at final follow-up (Balzer et al. 2018). Our center also treated two teenage patients with similar clinical manifestations in the past decade. However, in recent years, we found that more teenage lung cancers were incidentally diagnosed, small in size, GGO nodules in radiology, pre-invasive or invasive adenocarcinoma in pathology, early stage (Ia) with a good prognosis, as shown in the study. The cancers in these patients seemed indolent, as no progress was identified during the surveillance, and seemed not closely related to heredity, as only $3(25 \%)$ of them had family cancer history and none of their family members suffered from lung cancer. Our findings call for more attention to GGO featured lung adenocarcinoma in teenagers and urge to formulate a strategy to early diagnose and to early treat these patients.

Why do teenagers develop GGO featured lung tumor? In adult patients, tobacco is considered to be a main risk factor for lung cancer (Daff et al. 1951; Samet et al. 2009). However, only one patient in the study had a smoking history, that was only half pack a day for half a year. It has been reported that other factors, such as exposure to second-hand smoke, kitchen fumes or air pollution, may partly explain the incidence of GGO nodules in non-smokers (Samet et al. 2009). However, the amount and duration of exposure to these risk factors are probably not sufficient to lead to lung cancers in teenage patients. Further research in hereditary factors, such as germline mutation, may improve the understanding of pathogenesis in these lung cancers and help explain why teenagers develop lung cancer in such a young age.

Screening is probably an effective strategy for the detection of early-stage lung cancer in teenage patients, as the majority of that is asymptomatic and diagnosed incidentally according to the study. Some may deem that screening in the teenage population is unnecessary in consideration of "lead time bias". "Lead time bias" is a term that could be interpreted as although a disease is detected earlier by screening compared to usual clinical diagnosis, the person 
with the disease dies at the same time in life as those who were diagnosed later. In the study, we found that teenage patients could be cured by surgical resection when lung cancers were in early-stage, as no individual developed recurrence in follow-up. Thus, we guess that "lead time bias" doesn't work under the circumstances. On the other hand, the screening strategy for early-stage lung cancer in teenage patients remains unclear. The routine screening program for high-risk individual of lung cancer, annual LDCT, is probably not appropriate for teenage patients, because the potential risks of radiation exposure result from LDCT may vastly outweigh benefits (Bach et al. 2012). Therefore, we propose a screening strategy with long intervals based on a baseline CT scan, as a candidate strategy for the teenage population. If the baseline CT is negative, the interval to the next $\mathrm{CT}$ scan of the teenager could be five to ten years. The screening strategy is supported by the fact that most lung cancers in teenage patients are GGO featured early-stage cancers, which is characterized as slow-growing or indolent (Zhang et al. 2020). Even if they develop new suspected lung cancer during the long screening interval, the situations are probably manageable, and the prognosis should not be affected.

In the management of lung tumors in teenagers, 3-6 months of surveillance after initial detection of GGO nodules is generally essential as a benign lesion may disappear, while a persistent one is highly likely to be diagnosed as lung adenocarcinoma after resection (92.6\%) (Ye et al. 2018). For the persistent GGO nodules in teenage patients, we deem that surgery is preferable to surveillance. For surveillance, teenagers with GGO featured nodules should receive a CT scan every 1-2 years for 5 years or a surgical resection if nodules progress in size or solid component, according to Fleischner Society guidelines (MacMahon et al. 2017). However, Lee et al. proposed that GGO nodules could grow even when it is smaller than $6 \mathrm{~mm}$ and stable for 5 years (Lee et al. 2019), implying the progression time of the nodules is unpredictable. In this case, the surveillance would be long-term, even lifelong. Moreover, the follow-up after resection would be in a frequency of every 6 months performing CT scan if the lesion progresses into invasive adenocarcinoma, instead of an extended follow-up interval if the resection is performed in pre-invasive phase (Zhang et al. 2020). Thus, teenagers would accumulatively be exposed to a large dose of radiation during surveillance. On the other hand, the decision of surgery or surveillance should be made after thoughtful discussion between a patient, parents and doctors. Multidisciplinary discussion may also be required in the process (Zhang et al. 2021).

In short, GGO could be a sign of early-stage teenage lung adenocarcinoma. We proposed a screening strategy with long intervals based on a baseline CT scan for the teenage population, and a treatment strategy for diagnosed teenage patients.
Authors' contributions All authors listed in the manuscript have made substantial contributions to the completion of the study. HW and YZ were responsible for the acquisition and interpretation of data and drafting the manuscript. HH revised the content of the manuscript. YL and XS were responsible for the pathological analysis. QL and SW were in charge of radiological analysis. HC proposed the conception and designed the study.

Funding This work was supported by the National Natural Science Foundation of China (81930073), Shanghai Municipal Science and Technology Major Project (Grant No. 2017SHZDZX01, VBH1323001/026), Shanghai Municipal Key Clinical Specialty Project (SHSLCZDZK02104), and Pilot Project of Fudan University (IDF159034).

Code availability Not applicable.

Availability of data and material The datasets of the current study are available from the corresponding author on reasonable request.

\section{Declarations}

Conflict of interest The authors declare no conflict of interest.

\section{References}

Bach PB, Mirkin JN, Oliver TK et al (2012) Benefits and harms of CT screening for lung cancer: a systematic review. JAMA 307(22):2418-2429. https://doi.org/10.1001/jama.2012.5521

Balzer BWR, Loo C, Lewis CR, Trahair TN, Anazodo AC (2018) Adenocarcinoma of the lung in childhood and adolescence: a systematic review. J Thorac Oncol 13(12):1832-1841. https://doi.org/10. 1016/j.jtho.2018.08.2020

Daff ME, Doll R, Kennaway EL (1951) Cancer of the lung in relation to tobacco. Br J Cancer 5(1):1-20. https://doi.org/10.1038/bjc. 1951.1

Emori Y, Kiura K, Yoshino T et al (1999) Very young patient with peculiar squamous cell carcinoma of the lung. Intern Med 38(12):979-983. https://doi.org/10.2169/internalmedicine.38.979

Fu F, Zhang Y, Wen Z et al (2019) Distinct prognostic factors in patients with stage i non-small cell lung cancer with radiologic part-solid or solid lesions. J Thorac Oncol 14(12):2133-2142. https://doi.org/10.1016/j.jtho.2019.08.002

Jemal A, Miller KD, Ma J et al (2018) Higher lung cancer incidence in young women than young men in the United States. N Engl J Med 378(21):1999-2009. https://doi.org/10.1056/NEJMoa1715907

Lee HW, Jin KN, Lee JK et al (2019) Long-term follow-up of ground-glass nodules after 5 years of stability. J Thorac Oncol 14(8):1370-1377. https://doi.org/10.1016/j.jtho.2019.05.005

Luo X, Zheng S, Liu Q et al (2017) Should nonsmokers be excluded from early lung cancer screening with low-dose spiral computed tomography? Community-based practice in Shanghai. Transl Oncol 10(4):485-490. https://doi.org/10.1016/j.tranon.2017.02. 002

MacMahon H, Naidich DP, Goo JM et al (2017) Guidelines for management of incidental pulmonary nodules detected on CT images: from the Fleischner Society 2017. Radiology 284(1):228-243. https://doi.org/10.1148/radiol.2017161659

Polo V, Zago G, Frega S et al (2015) Non-small cell lung cancer in a very young woman: a case report and critical review of the literature. Am J Case Rep 16:782-789. https://doi.org/10.12659/ ajcr.894426 
Samet JM, Avila-Tang E, Boffetta P et al (2009) Lung cancer in never smokers: clinical epidemiology and environmental risk factors. Clin Cancer Res 15(18):5626-5645. https://doi.org/10.1158/10780432.CCR-09-0376

Tajiri T, Suita S, Shono K et al (1999) Lung cancer in a child with a substantial family history of cancer. Eur J Pediatr Surg 9(6):409412. https://doi.org/10.1055/s-2008-1072294

Ye T, Deng L, Xiang J et al (2018) Predictors of pathologic tumor invasion and prognosis for ground glass opacity featured lung adenocarcinoma. Ann Thorac Surg 106(6):1682-1690. https://doi. org/10.1016/j.athoracsur.2018.06.058

Zhang Y, Fu F, Chen H (2020) Management of ground-glass opacities in the lung cancer spectrum. Ann Thorac Surg 110(6):1796-1804. https://doi.org/10.1016/j.athoracsur.2020.04.094
Zhang Y, Jheon S, Li H et al (2020) Results of low-dose computed tomography as a regular health examination among Chinese hospital employees. J Thorac Cardiovasc Surg. 160(3):824-831. https://doi.org/10.1016/j.jtcvs.2019.10.145

Zhang Y, Chen Z, Hu H, Chen H (2021) Surgical strategies for preand minimally invasive lung adenocarcinoma 3.0: lessons learned from the optimal timing of surgical intervention. Semin Thorac Cardiovasc Surg. https://doi.org/10.1053/j.semtcvs.2020.12.009

Publisher's Note Springer Nature remains neutral with regard to jurisdictional claims in published maps and institutional affiliations. 